\title{
Efficient treatment of seven type 2 diabetes patients with human pluripotent stem cell transplantations overexpressing human insulin and/or ERR $\gamma$ and GLP- I genes
}

\begin{abstract}
Although daily injection of insulin (INS) is a common treatment for human diabetes, exogenous INS administration has little function to prevent the development of diabetes complications. Previously, we investigated the efficacies of transplantation of directly-generated human pluripotent stem cells (dgHPSCs) overexpressing human INS and/or estrogen-related receptor $\gamma(\mathrm{ERR} \gamma)$ genes for the treatment of human diabetes patients. In this study, we treated other seven human type 2 diabetes (T2D) cases with transplantation of dgHPSCs overexpressing INS, ERR $\gamma$ and glucagonlike peptide 1 (GLP-1) genes. Our data proved that this strategy can increase the secretion of C-peptide (C-P), decrease the patient's glucose (GLU) and HbA1c levels efficiently. To date, we treated 20 human T2D patients with our invented strategies. Thus, our method might lay a very important foundation for the eventually cure of human diabetes.
\end{abstract}

Keywords: dgHPSCs, ERR $\gamma$, INS, GLP-1, C-peptide, Human T2D, HbA1c
Volume 5 Issue I - 2019

\author{
Taihua Wang, ${ }^{1,2,3}$ Xiaojuan Diao, ${ }^{1,2}$ Rongrong \\ Li, ${ }^{1,2}$ Qingchang Fang, ${ }^{1,2}$ Xinyi Shi, ${ }^{3}$ Limin \\ Zhang, ${ }^{1,2}$ Guoke Yang, ${ }^{1,2}$ Ying Meng ${ }^{1,2}$ \\ IInterventional Hospital of Shandong Red Cross Society, \\ Shandong Province, China \\ ${ }^{2}$ Shandong New Medicine Research Institute of Integrated \\ Traditional and Western Medicine, Shandong Province, China \\ ${ }^{3}$ Cell Biotechnology Co., Ltd, Guangdong Province, China
}

Correspondence: Taihua Wang, Interventional Hospital of Shandong Red Cross Society, Jinan, Shandong Province, China, Email ganxibaowangtaihua@163.com

Received: January 16, 2019 | Published: January 22, 2019

\section{Introduction}

Human diabetes, including type 1 (T1D) and type 2 (T2D), are incurable diseases with current traditional treatment by exogenous daily human INS administration, although daily INS injection can do control the blood GLU levels during a long term. Yet, injection of INS only will eventually result in the development of diabetes complications. ${ }^{1,2}$ Recently, in two T2D patients, we reported that transplantation of dgHPSCs overexpressing human INS and/or ERR $\gamma$ genes could efficiently decrease the patient's blood GLU levels, improve the secretion of C-P, and reverse the diabetes complications, which brings the promising expectations for completely cure human T1D and T2D. ${ }^{3-5}$

GLP-1 is a 30 -amino acid peptide hormone produced in the intestinal epithelial endocrine L-cells by differential processing of proglucagon (PG) gene. ${ }^{6}$ The main actions of GLP-1 are to stimulate INS secretion, to inhibit glucagon secretion. GLP-1 also appears to be a physiological regulator of appetite and food intake, therefore, GLP1 is currently being evaluated for the therapy of T2D. ${ }^{6}$ Sequencing of the naturally occurring peptides extracted from human gut revealed that the structure of native GLP-1 corresponds to PG 78-107 amino acid sequence, and this truncated peptide, designated as GLP-1(7-36), was found to be a potent stimulator of GLU-induced INS secretion, whereas, the full-length GLP-1 was inactive. ${ }^{6-8}$

During the past year, besides these two reported T2D patients, ${ }^{3-5}$ we also treated many other T2D patients with similar strategies. All the treatments are similar with our reports earlier. ${ }^{3-5}$ The dgHPSCs numbers per transplantation are around $1 \times 10^{8}$ cells, overexpressing human INS and/or ERR $\gamma$, and human GLP-1 (7-36) genes, individually or simultaneously. ${ }^{3-5}$ Taken together, our reports are the first in the world to predict that human diabetes might be completely cured eventually via stem cell transplantation therapy.

\section{Statement of ethical approval}

The treatments for the patients and the use of human stem cells were approved by the Ethics Committee of Interventional Hospital of Shandong Red Cross Society (Shengjieyi 2003, No. 26) in compliance with Helsinki Declaration. The Ethics Committee of Interventional Hospital of Shandong Red Cross Society approved this clinical study and treatments. The participants provided their written confirmed consent to participate the clinical study and treatments. The Ethics Committee of Interventional Hospital of Shandong Red Cross Society approved this consent procedure. All the treatments for the patients and use of human stem cells were performed in accordance with the guidelines established in Interventional Hospital of Shandong Red Cross Society approved by the Ethics Committee. After traditional daily INS injection for several years, the patients agreed to try the stem cell therapy with overexpression of INS, ERR $\gamma$ and GLP-1(736) genes in our hospital to treat and cure their T2D disease. The stem cells used in these clinical treatments are dgHPSCs Lines stored at our Stem Cell Bank. All these stem cells were isolated and proliferated with the written confirmed consent of the participants, and all the culture, proliferation, transduction and preparation procedures of the stem cells were exactly the same as previously described. ${ }^{3-5}$

\section{Patient's treatment and results}

\section{Patient case \# I}

L-S S, male, aged 63, the comparison of his T2D symptoms before and after the stem cell transplantation therapy was listed in Table 1. From Table 1, we found that, after totally 9 times transplantations of dgHPSCs overexpressing human insulin/ERR $\gamma$ genes, the patient's fasting glucose (F-GLU) and glycosylated haemoglobin ( $\mathrm{HbA} 1 \mathrm{c})$ levels decreased significantly, and his C-P secretion increased obviously, and at the same time, his daily injection doses of INS were 
decreased greatly. These data demonstrated that our treatment method was very efficient for this patient.

\section{Patient case \#2}

L-T Z, male, aged 60, the comparison of his T2D symptoms before and after the stem cell transplantation therapy was listed in Table 2.

The data in Table 2 showed that, after totally 9 times of stem cell transplantations of dgHPSCs overexpressing human insulin/ ERR $\gamma$ genes, both the patient's F-GLU and HbA1c levels decreased significantly. As indicated in Table 2, after the third course of transplantations, both of the F-GLU and $\mathrm{HbAlc}$ levels are within the normal ranges. Moreover, his C-P secretion increased significantly, and at the same time, his daily injection doses of INS were decreased radically. These data are consistent with the patient case \#1, and demonstrated that our treatment method was very efficient.

Table I The comparison of T2D symptoms before and after the stem cell transplantation therapy of L-S S

\begin{tabular}{|c|c|c|c|c|}
\hline Stem cell transplantations & $\begin{array}{l}\text { F-GLU levels } \\
(3.9-6.1 \mathrm{mmol} / \mathrm{L})\end{array}$ & $\begin{array}{l}\text { HbA I c levels } \\
(4.2-6.3 \%)\end{array}$ & $\begin{array}{l}\text { C-P } \\
(0.37-1.47 \mathrm{nmol} / \mathrm{L})\end{array}$ & $\begin{array}{l}\text { INS injection } \\
\text { (IU/Day) }\end{array}$ \\
\hline Before transplantations & 15.5 & 9.4 & 0.76 & 38 \\
\hline After the first course of transplantations ( 3 times) & 10.3 & 9.1 & 0.76 & 30 \\
\hline After the second course of transplantations ( 3 times) & 8.24 & 8.2 & 0.76 & 22 \\
\hline After the third course of transplantations ( 3 times) & 7.9 & 8 & 0.81 & 10 \\
\hline
\end{tabular}

Table 2 The comparison of T2D symptoms before and after the stem cell transplantation therapy of L-T Z

\begin{tabular}{|c|c|c|c|c|}
\hline Stem cell transplantations & $\begin{array}{l}\text { F-GLU levels } \\
(3.9-6.1 \mathrm{mmol} / \mathrm{L})\end{array}$ & $\begin{array}{l}\text { HbA I c levels } \\
(4.2-6.3 \%)\end{array}$ & $\begin{array}{l}\text { C-P } \\
(0.37-1.47 \mathrm{nmol} / \mathrm{L})\end{array}$ & $\begin{array}{l}\text { Insulin injection } \\
\text { (IU/Day) }\end{array}$ \\
\hline Before transplantation & 10.04 & 10.06 & 1.37 & 44 \\
\hline After the first course of transplantations ( 3 times) & 7.69 & 7.75 & $\mathrm{~N} / \mathrm{A}$ & 28 \\
\hline After the second course of transplantations ( 3 times) & 8.29 & 7.29 & 1.56 & 22 \\
\hline After the third course of transplantations ( 3 times) & 4.22 & 6.4 & 1.72 & 15 \\
\hline
\end{tabular}

\section{Patient case \#3}

H-G T, male, aged 51, the comparison of his T2D symptoms before and after the stem cell transplantation therapy was listed in Table 3.

The results (Table 3) revealed that, after totally 9 times of stem cell transplantations of dgHPSCs overexpressing human insulin/
ERR $\gamma$ genes, the patient's F-GLU and HbAlc levels decreased significantly. Coincidentally, after three courses of transplantations, his C-P secretions also increased significantly. In addition, his daily takings of medicine, Metformin, were kept the same doses. These data demonstrated that our treatment method was very efficient as well.

Table 3 The comparison of T2D symptoms before and after the stem cell transplantation therapy of H-G T

\begin{tabular}{|c|c|c|c|c|}
\hline Stem cell transplantations & $\begin{array}{l}\text { F-GLU levels } \\
(3.9-6.1 \mathrm{mmol} / \mathrm{L})\end{array}$ & $\begin{array}{l}\text { HbA I c levels } \\
(4.2-6.3 \%)\end{array}$ & $\begin{array}{l}\text { C-P } \\
(0.37-1.47 \mathrm{nmol} / \mathrm{L})\end{array}$ & $\begin{array}{l}\text { Metformin } \\
\text { taking (tablets) }\end{array}$ \\
\hline Before transplantation & 9.14 & 10.4 & 0.62 & I \\
\hline After the first course of transplantations ( 3 times) & 7.4 & 13.2 & 0.86 & I \\
\hline After the second course of transplantations ( 3 times) & 10.52 & 9.29 & 0.93 & I \\
\hline After the third course of transplantations ( 3 times) & 7.02 & 6.5 & 1.72 & I \\
\hline
\end{tabular}

\section{Patient case \#4}

Y-S X, male, aged 65, the stem cell transplantation and the comparison of his T2D symptoms before and after the stem cell transplantation therapy was listed in Table $4 \& 5 .^{3-5}$

The results revealed that, after totally five times of stem cell transplantations of dgHPSCs overexpressing human insulin or ERR $\gamma$ genes (Table 4), the patient's F-GLU levels decreased generally from $11.68 \mathrm{mmol} / \mathrm{L}$ down to $7.31 \mathrm{mmol} / \mathrm{L}$, and the C-P secretion increased from $2.47 \mathrm{ng} / \mathrm{ml}$ up to $3.15 \mathrm{ng} / \mathrm{ml}$ (Table 5). Consequently, his $\mathrm{HbA} 1 \mathrm{c}$ levels decreased from $7.1 \%$ down to $6.6 \%$ (Table 5). These data suggested that our treatment method was also efficient for this patient.
Table 4 The date and times of stem cell transplantations of patient Y-S X

\begin{tabular}{ll}
\hline Transplantation dates & Transplantation stem cell types \\
\hline $30-07-2017$ & dgHPSCs + pWPI/INS \\
$04-08-2017$ & dgHPSCs + pWPI/INS \\
$20-08-2017$ & dgHPSCs + pWPI/ERR $\gamma$ \\
$11-09-2017$ & dgHPSCs + pWPI/ERR $\gamma$ \\
$25-09-2017$ & dgHPSCs + pWPI/ERR $\gamma$ \\
\hline
\end{tabular}


Table 5 The comparison of T2D symptoms before and after the stem cell transplantation therapy of patient $Y$-S $X$

\begin{tabular}{lllll}
\hline Test dates & C-P (0.8-3.8ng/ml) & F-INS (3-25 $\mathbf{m U} / L)$ & HbAlc (4.2-6.3\%) & F-GLU (3.9-6.I mmol/L) \\
\hline $2017-10-18$ & N/A & N/A & 6.7 & N/A \\
$2017-11-30$ & 2.47 & 21.74 & 7.0 & 8.30 \\
$2018-5-6$ & 3.22 & 33.94 & 7.1 & 9.71 \\
$2018-6-12$ & 7.18 & 92.2 & 6.6 & 11.68 \\
$2018-6-13$ & N/A & N/A & N/A & 7.9 \\
$2018-8-16$ & 3.15 & 25.53 & 6.9 & 7.31 \\
\hline
\end{tabular}

\section{Patient case \#5}

$\mathrm{Z} \mathrm{S}$, male, aged 55, the stem cell transplantation and comparison of his T2D symptoms before and after the stem cell transplantation therapy was listed in Table 6 \& Table $7 \cdot^{3-5}$ Our data showed that, after totally eleven times of stem cell transplantations of dgHPSCs overexpressing human INS and/or ERR $\gamma$ genes, and human GLP-1(7-
36) gene (Table 6), the patient's F-GLU levels decreased generally from $10.49 \mathrm{mmol} / \mathrm{L}$ down to $7.8 \mathrm{mmol} / \mathrm{L}$, and the C-P secretion increased from $0.65 \mathrm{ng} / \mathrm{ml}$ up to $1.53 \mathrm{ng} / \mathrm{ml}$ (Table 7). Consequently, his $\mathrm{HbAlc}$ levels decreased from $8.0 \%$ down to $6.7 \%$ (Table 7). These data suggested that our treatment method was also efficient for this patient.

Table 6 The date and times of stem cell transplantations of patient Z S

\begin{tabular}{llll}
\hline Numbers & Transplantation dates & Stem cell types & Cell numbers \\
\hline$\# 1$ & $05-08-2017$ & dgHPSCs + pWPI-INS $(30 \mathrm{ml})$ & $4.96 \times 10^{7}$ \\
$\# 2$ & $12-08-2017$ & dgHPSCs + pWPI-INS $(50 \mathrm{ml})$ & $1.41 \times 10^{8}$ \\
$\# 3$ & $09-09-2017$ & dgHPSCs + pWPI-ERR $\gamma(25 \mathrm{ml})$ & $6.48 \times 10^{7}$ \\
$\# 4$ & $15-09-2017$ & dgHPSCs + pWPI-ERR $\gamma(25 \mathrm{ml})$ & $7.2 \times 10^{7}$ \\
$\# 5$ & $28-10-2017$ & dgHPSCs + pWPI-ERR $\gamma(25 \mathrm{ml})$ & $5.39 \times 10^{7}$ \\
$\# 6$ & $29-10-2017$ & dgHPSCs + pWPI-INS + pWPI-ERR $\gamma(50 \mathrm{ml}+50 \mathrm{ml})$ & $1.28 \times 10^{8}$ \\
$\# 7$ & $19-12-2017$ & dgHPSCs + pWPI-GLP-I $(7-36)(50 \mathrm{ml})$ & $1.34 \times 10^{8}$ \\
$\# 8$ & $14-01-2018$ & dgHPSCs + pWPI-GLP-I $(7-36)(50 \mathrm{ml})$ & $1.17 \times 10^{8}$ \\
$\# 9$ & $29-03-2018$ & dgHPSCs + pWPI-ERR $\gamma(50 \mathrm{ml})$ & $6.75 \times 10^{7}$ \\
$\# 10$ & $11-07-2018$ (AM) & dgHPSCs + pWPI-ERR $\gamma(50 \mathrm{ml})$ & $6.7 \times 10^{7}$ \\
$\# 11$ & $11-07-2018$ (PM) & dgHPSCs + pWPI-ERR $\gamma(50 \mathrm{ml})$ & $8.01 \times 10^{7}$ \\
\hline
\end{tabular}

Table 7 The comparison of T2D symptoms before and after the stem cell transplantation therapy of patient Z S

\begin{tabular}{lllll}
\hline Test dates & C-P $(\mathbf{0 . 8 - 3 . 8 n g / m l )}$ & F- INS (3-25 mU/L) & HbAIc (4-6.3\%) & F-GLU (3.9-6.I mmol/L) \\
\hline $2017-9-22$ & 0.70 & 11.46 & 7.1 & 6.84 \\
$2017-11-9$ & 0.65 & 13.51 & 7.9 & 8.32 \\
$2017-12-1$ & 0.98 & 17.27 & 8.0 & 9.24 \\
$2018-3-2$ & 0.90 & 10.12 & 7.5 & 10.1 \\
$2018-4-28$ & 0.91 & 14.95 & 7.6 & 9.92 \\
$2018-6-15$ & 0.76 & 13.46 & 8.0 & 10.49 \\
$2018-8-2 \mid$ & 1.53 & 13.44 & 6.7 & 7.8 \\
\hline
\end{tabular}

\section{Patient case \#6}

H-P L, female, aged 64, the stem cell transplantation and comparison of her T2D symptoms before and after the stem cell transplantation therapy was listed in Table 8 \& Table $9 .{ }^{3-5}$

These results demonstrated that, after totally 16 times of stem cell transplantations of dgHPSCs overexpressing human INS or ERR $\gamma$ genes, and human GLP-1(7-36) gene (Table 8), respectively, the patient's F-GLU levels decreased significantly from $18.88 \mathrm{mmol} / \mathrm{L}$ down to $4.12 \mathrm{mmol} / \mathrm{L}$, and the F-INS secretion increased slightly from $22.89 \mu \mathrm{U} / \mathrm{mL}$ to $23.95 \mu \mathrm{U} / \mathrm{mL}$ (Table 9 ). These data suggested that our treatment method was efficient for the patient. 
Table 8 The date and times of stem cell transplantations of patient H-P L

\begin{tabular}{|c|c|c|c|}
\hline Numbers & Transplantation dates & Stem cell types & Cell numbers \\
\hline$\# \mathbf{H}$ & $02-04-2017$ & dgHPSCs + pWPI-ERR $\gamma(5 \mathrm{ml})$ & $8 \times 10^{6}$ \\
\hline$\# 2$ & $24-04-2017$ & dgHPSCs + pWPI-ERR $\gamma(25 \mathrm{ml})$ & $3.83 \times 10^{7}$ \\
\hline \#3 & $05-06-2017$ & dgHPSCs + pWPI-INS $(20 \mathrm{ml})$ & $3.9 \times 10^{7}$ \\
\hline$\# 4$ & $09-06-2017$ & dgHPSCs + pWPI-INS (20 ml) & $4.28 \times 10^{7}$ \\
\hline$\# 5$ & $17-06-2017$ & dgHPSCs + pWPI-INS (40 ml) & $2.25 \times 10^{7}$ \\
\hline \#6 & $25-06-2017$ & dgHPSCs + pWPI-INS (40 ml) & $2.7 \times 10^{7}$ \\
\hline \#7 & $|I-07-20| 7$ & dgHPSCs + pWPI-INS (40 ml) & $1.16 \times 10^{8}$ \\
\hline \#8 & $12-07-20 \mid 7$ & dgHPSCs + pWPI-INS (40 ml) & $1.34 \times 10^{8}$ \\
\hline \#9 & $13-07-20 \mid 7$ & dgHPSCs + pWPI-INS $(40 \mathrm{ml})$ & $1.84 \times 10^{8}$ \\
\hline$\# 10$ & $20-08-2017$ & dgHPSCs + pWPI-INS (25 ml) & $5.8 \times 10^{7}$ \\
\hline \#II & $10-10-2017$ & dgHPSCs + pWPI- ERR $\gamma(25 \mathrm{ml})$ & $3.15 \times 10^{7}$ \\
\hline$\# 12$ & $16-10-2017$ & dgHPSCs + pWPI- ERR $\gamma(50 \mathrm{ml})$ & $9.88 \times 10^{7}$ \\
\hline$\# 13$ & $17-10-2017$ & dgHPSCs + pWPI- ERR $\gamma(25 \mathrm{ml})$ & $8.0 \times 10^{7}$ \\
\hline$\# 14$ & $13-12-2017$ & dgHPSCs + pWPI-GLP-I (7-36) (50 ml) & $1.33 \times 10^{8}$ \\
\hline$\# 15$ & $14-12-20 \mid 7$ & dgHPSCs + pWPI-GLP-I (7-36) (25 ml) & $1.04 \times 10^{8}$ \\
\hline$\# 16$ & $26-12-2017$ & dgHPSCs + pWPI-GLP-I (7-36) (50 ml) & $6.84 \times 10^{7}$ \\
\hline
\end{tabular}

Table 9 The comparison of T2D symptoms before and after the stem cell transplantation therapy of patient H-P L

\begin{tabular}{lll} 
Test date & F-GLU (3.9-6.I mmol/L) & F-INS (2.60-24.90 $\mu \mathrm{U} / \mathrm{mL})$ \\
\hline $2017-02-18$ & 18.88 & N/A \\
$2017-04-11$ & 13.13 & 22.89 \\
$2017-06-20$ & 4.12 & 23.95 \\
$2017-07-27$ & 12.54 & N/A
\end{tabular}

\section{Patient case \#7}

$\mathrm{J}$ F, female, aged 51, the stem cell transplantation and comparison of her T2D symptoms before and after the stem cell transplantation therapy was listed in Table $10 \& 11 .^{3-5}$ These results demonstrated that, after totally 8 times of stem cell transplantations of dgHPSCs overexpressing human INS and/or ERR $\gamma$ genes, (Table 10), respectively, the patient's F-INS secretion levels increased significantly from $5.99 \mu \mathrm{U} / \mathrm{mL}$ to $9.27 \mu \mathrm{U} / \mathrm{mL}$ (Table 11) and her HbAlc levels decreased from $8.2 \%$ to $7.7 \%$. These data showed that our treatment method was also efficient for the patient. Besides, we still have 11 other patient cases, who were treated with the similar strategy and all of them also revealed positive improvements for their T2D symptoms (Data not shown).

Table I0 The date and times of stem cell transplantations of patient J F

\begin{tabular}{llll}
\hline Numbers & Transplantation dates & Stem cell types & Cell numbers \\
\hline$\# 1$ & $25-06-2017$ & dgHPSCs + pWPI-INS $(25 \mathrm{ml})$ & $2.7 \times 10^{7}$ \\
$\# 2$ & $29-06-2017$ & dgHPSCs + pWPI-INS $(40 \mathrm{ml})$ & $3.34 \times 10^{7}$ \\
$\# 3$ & $05-07-2017$ & dgHPSCs + pWPI-ERR $\gamma+I N S(30 \mathrm{ml}+30 \mathrm{ml})$ & $9.0 \times 10^{7}$ \\
$\# 4$ & $09-08-2017$ & dgHPSCs + pWPI-ERR $\gamma+I N S(50 \mathrm{ml}+50 \mathrm{ml})$ & $1.0 \times 10^{8}$ \\
$\# 5$ & 2017.8 .21 & dgHPSCs + pWPI-ERR $\gamma+I N S(50 \mathrm{ml}+50 \mathrm{ml})$ & $3.56 \times 10^{7}$ \\
$\# 6$ & $05-09-2017$ & dgHPSCs + pWPI-ERR $(25 \mathrm{ml})$ & $4.6 \times 10^{7}$ \\
$\# 7$ & $09-10-2017$ & dgHPSCs + pWPI-ERR $\gamma(25 \mathrm{ml})$ & $5.0 \times 10^{7}$ \\
$\# 8$ & $10-10-2017$ & dgHPSCs + pWPI-ERR $\gamma(25 \mathrm{ml})$ & $3.15 \times 10^{7}$ \\
\hline
\end{tabular}

Table II The comparison of T2D symptoms before and after the stem cell transplantation therapy of patient J F

\begin{tabular}{|c|c|c|}
\hline Test date & HbAlc (\%) & F-INS $(2.60-24.90 \mu \mathrm{U} / \mathrm{mL})$ \\
\hline II-08-2017 & 8.2 & 5.99 \\
\hline $06-10-2017$ & 7.7 & 9.27 \\
\hline
\end{tabular}

\section{Discussion}

It is reported that approximately $25.9 \%$ of Americans of 65 years or older have diabetes, whereas, $9.3 \%$ of those in the general population. ${ }^{2}$ Yet, so far, the only efficient method to control the patient's blood GLU levels is to inject exogenous INS, but the daily administration of INS cannot prevent from the development of 
diabetes complications. ${ }^{1}$ Generally, besides the control of blood GLU levels within the physiological range by daily INS administration, there are two other more important aims must be achieved in order to completely cure human diabetes, which are to repair the functions of pancreatic $\beta$ cells to improve the secretion of INS and to restore and even reverse the developed diabetes complications. ${ }^{5}$ To date, the only successful investigations are performed in mouse models, and the data revealed that the transplanted differentiated human pancreatic $\beta$ cells could incorporate into the recipients and repair their pancreatic $\beta$ functions. ${ }^{9,10}$ Recently, we reported that transplantations of dgHPSCs overexpressing human INS and/or ERR $\gamma$ genes can not only decrease the patient's blood GLU levels, but also can increase the secretion of C-P, and even can repair and reverse the developed diabetic complications. ${ }^{3-5}$ Therefore, our investigations shed promising light on the completely cure of human T2D disease with very positive expectations.

In this clinical study, we reported seven other patient cases, who were treated with the similar strategies for their T2D. Surprisingly, all of them showed significant improvement of their T2D symptoms, including the decrease of blood GLU levels and INS daily injection doses, the increase of C-P secretion, etc. In addition, we also treated T2D patients with dgHPSCs overexpressing human GLP-1(7-36) gene, which is a well-known gene with the functions to stimulate INS secretion, and to inhibit glucagon secretion. ${ }^{6}$ Our data showed that dgHPSCs-GLP-1(7-36) also can be employed for the treatment of human T2D (Table 6) (Table 8).

In our previous reports, ${ }^{3-5}$ we reported two T2D patient cases treated with the transplantations of dgHPSCs overexpressing human INS and/or ERR $\gamma$ genes. Besides the seven T2D patient cases reported here, we still have 11 other patient cases, who were also currently under treatment with the similar strategy and all of them revealed positive improvements for their T2D symptoms (Data not shown). Taken together, totally, we already treated 20 T2D patients with our newly invented strategies clinically, all of them showed significant improvement of their diabetic symptoms. Our investigations laid very promising foundations for eventually cure human diabetes in the near future.

\section{Authors contributions}

T W instructed and supervised the whole clinical work. R L, Q F, $\mathrm{X}$ D, L Z, G Y and Y M worked on the culture and clinical treatments of the cells. All the authors discussed, wrote, read and approved the final manuscripts.

\section{Acknowledgments}

The authors wish to thank the nurses in our hospital for their help and caring of the patients during the clinical treatments.

\section{Conflicts of interest}

Author declares that there is no conflicts of interest.

\section{References}

1. Minami K, Seino S. Current status of regeneration of pancreatic $\beta$-cells. J Diabetes Investig. 2013;4:131-141.

2. Palmer AK, Tchkonia T, LeBrasseur NK, et al. Cellular senescence in Type 2 Diabetes: a therapeutic opportunity. Diabetes. 2015;64:22892298.

3. Wang T, Wang X, Zhang B, et al. Transplantation of human pluripotent stem cells overexpressing ERR $\gamma$ can efficiently improve the symptoms of type 2 diabetes patient. Adv Tissue Eng Regen Med Open Access. 2018;4(3):457-462.

4. Wang T, Chen X, Cui X, et al. Transplantation of human pluripotent stem cells overexpressing insulin/ERR $\gamma$ can efficiently decrease the HbA1c levels of type 2 diabetes patient (Case \#2-A). International Journal of Current Research. 2018;10(10):74690-74696.

5. Wang T, Wang X, Zhang B, et al. Transplantation of Human dgHPSCs Overexpressing Insulin and ERR $\gamma$ can Efficiently Decrease the Glucose and HbA1c levels, Increase the Secretion of C-peptide and Repair the Complications of Coronary Heart Disease in T2D Patient (Case \#1-A). Stem Cells Regen Med. 2018;2(2):1-9.

6. Holst JJ. The physiology of glucagon-like peptide 1. Physiol Rev. 2007;87:1409-1439.

7. Holst JJ, Orskov C, Nielsen OV, et al. Truncated glucagon-like peptide I, an insulin-releasing hormone from the distal gut. FEBS Lett. 1987;211:169-174

8. Mojsov S, Weir GC, Habener JF. Insulinotropin: glucagon-like peptide I (7-37) co-encoded in the glucagon gene is a potent stimulator of insulin release in the perfused rat pancreas. J Clin Invest. 1987;79:616-619.

9. Pagliuca FW, Millman JR, Gürtler M, et al. Generation of functional human pancreatic $\beta$ cells in vitro. Cell. 2014;159:428-439.

10. Yoshihara E, Wei Z, Lin CS, et al. ERR $\gamma$ is required for the metabolic maturation of therapeutically functional glucoseresponsive $\beta$ cells. Cell Metabolism. 2016;23:622-634. 